\title{
A PSICOLOGIA NA UNIVERSIDADE ABERTA À TERCEIRA IDADE: EXPERIÊNCIAS DE ATUAÇÃO COM IDOSOS NA UNATI/UNESP, CAMPUS DE ASSIS
}

\section{THE PSYCHOLOGY AT THE OPEN UNIVERSITY TO THIRD AGE: EXPERIENCES OF ACTION WITH ELDERLY PEOPLE IN UNATI / UNESP, CAMPUS DE ASSIS}

\author{
PSICOLOGÍA EN LA UNIVERSIDAD ABIERTA PARA ADULTOS MAYORES: \\ EXPERIENCIAS CON ANCIANOS EN LA UNATI / UNESP, CAMPUS ASSIS
}

Mariele Rodrigues Correa*

\begin{abstract}
Resumo: Este trabalho relata a experiência de duas atividades desenvolvidas pelo curso de Graduação em Psicologia, junto a idosos inscritos no projeto "Universidade Aberta à Terceira Idade", da Universidade Estadual Paulista, campus de Assis, a saber, uma com idosos institucionalizados e outra com pessoas da terceira idade. O objetivo dessas atividades é fomentar estratégias de intervenção em grupos, no formato de oficinas temáticas baseadas no referencial de grupo operativo, que possam produzir expansão da subjetividade dos idosos, de maneira a combater o isolamento social e também promover (re)significações do processo de envelhecer. São realizadas oficinas semanalmente com uma hora e meia de duração, coordenadas por um grupo de treze estagiários do curso de Psicologia, inscritos no Estágio Curricular "Envelhecimento e Processos de Subjetivação", sob nossa supervisão. Os grupos contam com a participação de aproximadamente 40 idosos. Essa experiência, que caminha para seu terceiro ano, tem possibilitado criar novas ferramentas de atuação em Psicologia para além do modelo clínico tradicional. Outro aspecto a se destacar é que os grupos tem se mostrado como um lugar estratégico de empoderamento no processo de envelhecer, uma vez que o dispositivo grupal favorece trocas simbólicas, diferentes olhares para temáticas abordadas e assimilação de diversos processos de subjetivação. Observamos que, quando são estimulados, eles redescobrem sua capacidade de refletir, criticar e ponderar sobre diversos assuntos, podendo produzir processos de subjetivação mais potencializados nos encontros com o outro. Além disso, o fato de as oficinas serem coordenadas por graduandos favorece e fomenta, positivamente, as relações e trocas intergeracionais.
\end{abstract}

Palavras-chave: Psicologia. Grupos. Subjetividade. Idosos.

Abstract: This work reports the experience of two activities developed by the Psychology Undergraduate course, along with the elderly enrolled in the project "Open University to the Third Age", from the State University of São Paulo, Assis campus, namely, one with institutionalized elderly people and another with people of old age. The goal of these activities is to encourage intervention strategies in groups, in the form of thematic workshops based on the operational group referral, that can produce an expansion of the subjectivity of the elderly, in order to combat social isolation and also promote (re)meanings of the aging process. Weekly workshops with an hour and a half duration are held, coordinated by a group of thirteen trainees from the Psychology course, enrolled in the Curricular Internship "Aging and Subjectivation Processes", under our supervision. The groups rely on the participation of approximately 40 elderly people. This experience, which is in its third year, has made it possible to create new tools

\footnotetext{
* Doutora em Psicologia e Sociedade pela Universidade Estadual Paulista "Júlio de Mesquita Filho" (UNESP), campus de Assis. Professora do Departamento de Psicologia Evolutiva, Social e Escolar da FCL - UNESP, campus de Assis. E-mail: mariele@assis.unesp.br
} 
for acting in Psychology beyond the traditional clinical model. Another aspect to emphasize is that the groups have been shown as a strategic place of empowerment in the process of aging, since the group device favors symbolic exchanges, different views on the topics addressed and assimilation of several processes of subjectivation. We observe that when they are stimulated, they rediscover their ability to reflect, criticize and ponder on various subjects, and may produce subjectivation processes that are more enhanced in the meetings with the other. In addition, the fact that the workshops are coordinated by graduates positively favors and fosters intergenerational relations.

Keywords: Psychology. Groups. Subjectivity. Elderly.

Resumen: Este artículo relata la experiencia de dos actividades desarrolladas por el curso de Graduación en Psicología, con los ancianos inscriptos en el proyecto "Universidad Abierta de la Tercera Edad", de la Universidade Estadual Paulista, campus de Assis: una con ancianos institucionalizados y otra con personas de la tercera edad. El objetivo de estas actividades es promover estrategias de intervención en grupos, en forma de talleres temáticos basados en grupo operativo, para producir la expansión de la subjetividad de las personas mayores con el fin de combatir el aislamiento social y promover el proceso de (re)significación de envejecimiento. Se realizan talleres semanales de una hora y media, coordinados por un grupo de trece pasantes del curso de Psicología, inscriptos en el espacio curricular "Envejecimiento y Procesos de Subjetivación" bajo nuestra supervisión. Los grupos cuentan con la participación de aproximadamente 40 personas de edad avanzada. Esta experiencia, que ya va para su tercer año, ha hecho posible la creación de nuevas herramientas de acción en psicología más allá del modelo clínico tradicional. Otro aspecto a destacar es que los grupos aparecen como un lugar estratégico de empoderamiento en el proceso de envejecimiento, ya que el grupo, como dispositivo, favorece los intercambios simbólicos, diversas miradas hacia los temas abordados y la asimilación de los diversos procesos subjetivos. Hemos observado que, al ser estimulados, redescubren su capacidad de reflexionar, criticar y reflexionar sobre diversos temas y pueden producir procesos subjetivos más potentes en los encuentros con el otro. Además, el hecho de que los talleres son coordinados por estudiantes de pregrado favorece y estimula positivamente las relaciones y los intercambios intergeneracionales

Palabras claves: Psicología. Grupos. Subjetividad. Adulto Mayor.

\section{Introdução}

Ao longo das últimas décadas, diversas pesquisas tem apontado o crescente processo de envelhecimento populacional. No Brasil, por exemplo, a expectativa de vida elevou consideravelmente seus índices e a proporção de idosos aumentou sobremaneira, chegando a ultrapassar alguns países europeus, em números absolutos. (MINAYO, 2011). Tal conquista é, sem dúvida, importante indicador da melhoria na qualidade de vida da população brasileira.

Além disso, o aumento do número de idosos também provoca algumas interessantes mudanças na maneira que nosso país normalmente se representa. É muito comum, especialmente há poucas décadas atrás, dizer-se que o Brasil era um "país jovem”. Se esse era o discurso rotineiro, arriscaríamos dizer que, no futuro próximo - poderíamos até dizer no presente mesmo - seremos um país de cabelos grisalhos. (VERAS, 1994). $\mathrm{O}$ avanço da ciência, a queda da taxa de natalidade, o acesso a bens de saúde e diversos outros fatores tem contribuído para que se tenha mais anos de vida e uma presença mais acentuada dos idosos no cenário social, gerando algumas discussões e problemáticas. (CAMARANO, 2009; CORREA; FRANÇA; HASHIMOTO, 2010). 
O envelhecimento populacional é uma conquista importante, que traz alguns desdobramentos e desafios a fim de fomentar e preservar a qualidade de vida nas idades avançadas. Nesse sentido, além de questões de suma importância a serem debatidas e implementadas, por exemplo, nas políticas públicas, também a ciência e, em particular, a Psicologia pode trazer diversas contribuições que enriqueçam a rede de cuidados e atenção à pessoa idosa.

Temos observado, nos últimos anos, que a Psicologia tem atendido à demanda da população de idade avançada em diversos espaços, como na prática clínica convencional e em diversos equipamentos relacionados às políticas públicas, especialmente nos serviços de saúde, de saúde mental e na assistência social. (CONSELHO FEDERAL DE PSICOLOGIA, 2008). Também, aos poucos, a ciência psi tem se feito notar na atenção à população institucionalizada em asilos. (GRAEFF, 2005; MAIA; CASTRO; JORDÃO, 2010; RAMOS, 2012).

Assim, na formação profissional, cada vez mais se tem demandado um preparo teórico-prático dos alunos para compreensão dos aspectos biopsicossociais do envelhecer para melhor acolher essa crescente demanda. Dessa maneira, em nosso curso de Graduação em Psicologia da Faculdade de Ciências e Letras da UNESP (Universidade Estadual Paulista), campus de Assis, trabalhamos em parceria direta com o projeto da Universidade Aberta à Terceira Idade (UNATI) de nosso campus, uma vez que se trata de um importantíssimo espaço de trocas e relações intergeracionais, além de possibilitar diversas aprendizagens aos alunos graduandos do curso.

Nossa entrada no projeto da UNATI se faz por meio um trabalho com grupos de idosos. Entendemos que, dos diversos dispositivos e práticas em Psicologia junto à população idosa, o atendimento em grupos se mostra como importante estratégia de sociabilidade e de trocas simbólicas e afetivas. A valorização da experiência grupal no envelhecimento pode ser uma estratégia de enfrentamento da condição de isolamento e de desqualificação do idoso, muitas vezes encontrada no meio social e familiar. A vivência da grupalidade pode propiciar uma experiência genuína e ímpar de pertencimento, de resgate do gregarismo, de solidariedade e companheirismo. É, ainda, uma possibilidade de construção de si no plano coletivo, uma experiência de envelhecer que abraçaria e promoveria formas múltiplas e singulares de devir-envelhecimento, nas fendas do isolacionismo, individualismo, privativismo e da solidão que se fortalecem o cenário contemporâneo.

Por isso, diante dos desafios que o envelhecimento da população lança para a sociedade e, mais particularmente à Psicologia como ciência e profissão, destacamos a necessidade de se criar estratégias de atuação com idosos que promovam expansão dos vínculos e a produção de subjetividade em contexto grupal.

No presente trabalho, discorreremos sobre a participação do curso de graduação em Psicologia no projeto da UNATI da UNESP, campus de Assis, destacando os grupos com os quais trabalhamos. O projeto da nossa UNATI se caracteriza como atividade de extensão e está vinculado, consequentemente, à Pró-Reitoria de Extensão. No campus de Assis, já se vão pouco mais de 20 anos de funcionamento da UNATI, com expressivo crescimento na participação da terceira idade a cada ano.

\section{Objetivos e metodologia}

O objetivo deste trabalho é relatar a experiência do curso de Graduação em Psicologia em sua atuação junto à Universidade Aberta 
à Terceira Idade (UNATI) da Universidade Estadual Paulista "Júlio de Mesquita Filho" (UNESP), campus de Assis. Diante da demanda de formação profissional para atenção em Psicologia com idosos, nos lançamos, há três anos, no propósito de desenvolver duas atividades em grupos para pessoas com idade superior a 60 anos, tanto para aquelas comumente caracterizadas como pertencentes à terceira idade (com envelhecimento ativo e independente), quanto para idosos institucionalizados da cidade de Assis (SP).

Atualmente, a UNATI da FCL(UNESP) de Assis conta com 332 idosos, em sua maioria mulheres. A programação do projeto conta com 34 oficinas/cursos e envolve uma série de atividades oferecidas semanalmente que estão relacionadas a aspectos artístico-culturais e à promoção de saúde, como aulas de yoga, ginástica meditativa, alimentação saudável, artesanato, dança, capoeira, seresta e outras. Também são oferecidas atividades voltadas para aprendizagem e trocas de experiências, como aulas de línguas estrangeiras, informática, letramento, jogos da mente (oficinas de memória) e as oficinas oferecidas pelo curso de Psicologia. De acordo com os dados oferecidos pela administração do projeto, muitos dos idosos participam de mais de uma atividade, o que resulta em 794 matrículas. A coordenação é de responsabilidade da atual vice-diretora da unidade e de uma servidora técnico-administrativa.

É importante destacar que, enquanto projeto de extensão da UNESP, toda a programação da UNATI de Assis é oferecida gratuitamente aos idosos da cidade e da região. Além disso, as oficinas e cursos são ministrados por servidores técnico-administrativos, docentes, alunos de diferentes cursos de graduação e pessoas da comunidade, o que contribui deveras para a promoção de encontros intergeracionais entre diferentes segmentos.
Dentro desta vasta programação oferecida pelo projeto da UNATI de Assis, destacamos, portanto, no presente trabalho, duas atividades desenvolvidas pelo núcleo de estágio profissionalizante do curso de Psicologia "Envelhecimento e Processos de Subjetivação", que são as "Oficinas de Psicologia com Idosos Asilados" e o grupo "Encontros com a Terceira Idade". O objetivo dessas atividades é fomentar estratégias de intervenção no formato de grupos que possam produzir a expansão da subjetividade dos idosos, de maneira a combater o isolamento social a que muitos estão submetidos e também de promover (res)significações do processo de envelhecer.

Para tanto, oferecemos duas atividades semanais distintas, no formato de oficinas e com o referencial de grupo operativo (PICHON-RIVIÈRE, 1983), com uma hora e meia de duração cada uma, na grade de programação do projeto da UNATI. Uma delas é a "Oficinas de Psicologia com Idosos Asilados", que conta com a participação de cerca de 20 idosos provenientes de duas instituições asilares da cidade de Assis (SP). Nessas oficinas desenvolvemos atividades de senso-percepção e expansão da mobilidade, além de encontros que buscam resgatar memórias e histórias de vida dos asilados e atividades que promovam a sociabilidade entre eles, tanto no espaço do campus como nos espaços da cidade. Já o grupo "Encontros com a Terceira Idade" tem cerca de 30 participantes com idade superior a 60 anos, majoritariamente composto por mulheres. Nesse espaço grupal, são discutidas diversas temáticas referentes ao processo de envelhecer e seu impacto na construção da subjetividade, como questões de gênero, família, sentidos e sentimentos do corpo e outros.

Entendemos que o referencial de grupo operativo oferece importantes ferramentas teórico-práticas para a elaboração das oficinas 
e também para o manejo das questões que emergem no grupo. De acordo com PichonRivière (1983), o principal objetivo de um grupo é que ele seja um espaço para reflexão e rompimento de esteriotipias, a fim de promover um processo de aprendizagem. Para o autor, a aprendizagem é um processo contínuo em que comunicação e interação são indissociáveis, na medida em que aprendemos a partir da relação com os outros - a partir dessa relação com o outro, com o diferente, para saber construir outros olhares e sentidos para as experiências da vida. Portanto, ao pensarmos no contexto da UNATI, tal aporte teórico possibilita trabalhar, junto ao grupo, processos de aprendizagens no contexto grupal e também na relação com os estagiários que coordenam as oficinas.

Vale assinalar que somos responsáveis pelo núcleo de estágio enquanto supervisora e as atividades oferecidas aos idosos são realizadas por estudantes do quarto e quinto anos do curso de graduação em Psicologia da Faculdade de Ciências e Letras (FCLUNESP), campus de Assis. Como docente do curso de Psicologia e responsável pelo desenvolvimento do referido núcleo de estágio, procuramos propiciar aos estagiários do curso de Psicologia da UNESP de Assis uma formação que lhes permitam articular a produção de conhecimento com a construção de novas estratégias de atuação profissional com essa população. Todas as atividades desenvolvidas junto aos idosos são discutidas e elaboradas previamente em supervisão semanal, com duração de quatro horas. Nessas reuniões, também são realizados seminários de estudo sobre Gerontologia e Psicologia do Envelhecimento, a fim de subsidiar nossa prática.

\section{Resultados}

Ao longo desses três anos de trabalho junto à UNATI de nosso campus, pudemos constatar que o grupo, como dispositivo de trabalho em nossa área, tem se mostrado como importante ferramenta de intervenção em Psicologia para expansão de vínculos e de enfrentamento de solidão e isolamento social, especialmente no caso dos idosos institucionalizados. Ao trabalharmos no formato de oficinas, também notamos que há a possibilidade de criar estratégias de atuação de maneira dinâmica e inovadora, abordando temáticas diversas referentes ao envelhecimento humano e à subjetividade.

Como as oficinas têm um funcionamento flexível e mais aberto, especialmente em termos de espaços físicos para se trabalhar em grupo, percebemos que os grupos cultivam e mantêm um forte vínculo com a comunidade acadêmica, por exemplo, na presença dos mais velhos em diferentes espaços institucionais, em conversas nos corredores com os estudantes e outros, o que contribui para que os idosos realmente se sintam parte da universidade e para que se fortaleçam as relações intergeracionais.

No caso do grupo "Encontros com a Terceira Idade”, as oficinas são desenvolvidas por quatro discentes de nosso núcleo de estágio, junto a um grupo de cerca de trinta idosas da UNATI, em uma sala de aula do prédio do curso de Psicologia. O objetivo principal desses encontros, que ocorrem semanalmente, é promover um espaço de reflexão grupal sobre o envelhecimento e seus desdobramentos no processo de subjetivação. Por isso, a reflexão e o compartilhamento de diferentes olhares e pontos de vista contribuem para a construção de sentidos diversos para o envelhecer, auxiliando na ruptura de esteriotipias. 
Nas oficinas, a cada semana trabalhamos com uma temática discutida e elaborada previamente nas supervisões. Os temas abordados são escolhidos pelo núcleo do estágio ou a partir de demandas do próprio grupo. Ao longo de três anos de desenvolvimento desse grupo, foi possível abordar diversos temas, como as representações acerca do envelhecimento (ser idoso/terceira idade), aspectos da vida, da sociedade e do próprio processo de envelhecimento, como a memória, senso-percepção, sexualidades e gêneros, histórias e projetos de vida, relações familiares, relações intergeracionais, avosidade, questões da atualidade, direitos do idoso, entre outros. Para abordar essas temáticas, utilizamos alguns disparadores, como uma música, um poema, imagens, vídeos ou outros dispositivos. Também procuramos produzir registros das atividades do grupo, por exemplo, em cartazes, folhas, fotografias, desenhos ou escritos.

A reflexão sobre o próprio envelhecer trouxe muitas questões complexas e bastante interessantes. No início de nosso grupo, ainda em 2013, havia muita resistência a termos como "idoso" e, principalmente, "velho". Dificilmente as mulheres participantes do grupo se posicionam como idosas e, menos ainda, como velhas. Boa parte delas entende que o termo "velho" se refere a algo inútil, desgastado e usado, o que não representaria a forma como elas se veem e se posicionam diante do mundo. Uma das participantes disse, em uma das ocasiões, que "velho é como um pano de chão gasto, que não serve para nada, nem para limpar”. Muitas das mulheres diziam preferir a expressão "juventude experiente" para se denominarem, pois afirmavam que se sentiam jovens e dinâmicas, porém, com muita bagagem e experiência de vida. Por isso, diziam preferir não voltar aos tempos de adolescência e juventude, pois ali não tinham o conhecimento e a sabedoria acumulada nos anos de vida.
A designação de "juventude experiente” para nós é muito significativa, pois congrega aspectos bastante diversos sobre a forma com que os idosos se percebem. Por um lado, há a valorização da juventude, do espírito jovem, do signo da atividade e do dinamismo. Por outro, destacam o aspecto da sabedoria, da maturidade psíquica, da pessoa mais velha como sendo aquela portadora de conhecimento. Não deixa de ser uma composição variada, de um corpo que comporta velocidade e tempo. Também nos chama atenção o quanto as palavras ganham sentidos diversos ao longo da história e da cultura. Como destaca Peixoto (1998), na França, no século XIX, o termo "velho" ou "velhote" se referia a indivíduos de idade elevada que não possuíam status social e sua conotação não era fortemente pejorativa, como nos dias atuais. O termo "idoso", por sua vez, era atribuído àqueles que se situavam nas camadas sócio-econômicas mais elevadas, sendo que, em meados do século passado, emerge a categoria de "terceira idade". Certamente estamos fazendo um resumo extremamente breve de uma história repleta de múltiplos sentidos e acontecimentos, mas interessa-nos destacar a dinamicidade das significações do envelhecer e o quanto esse processo histórico se compõe com a cultura, com a sociedade e com as vivências individuais, compondo tramas complexas e diversas facetas para o envelhecer.

Diversas outras temáticas abordadas também foram importantes para a constituição do grupo, como família (histórias e memórias familiares), questões de gênero (problematizando o papel das figuras femininas e masculinas ao longo das últimas décadas) e preconceitos (relacionados à raça, opção sexual, gênero e idade). Também trabalhamos com as datas comemorativas, como o amor e a diversidade das formas de amar na semana do dia dos namorados; os sentidos da filiação, procurando resgatar histórias e memórias dos 
participantes diante da condição de ser filho, na semana do dia das mães; e o tema trabalho, abordando o sentido deste, as profissões de cada um e a aposentadoria na semana de $1^{\circ}$ de maio, data em que se celebra o Dia do Trabalho.

Já nas Oficinas de Psicologia com Idosos Asilados, um dos primeiros desafios foi o de criar possibilidades de atuação com um grupo que apresentava diferentes graus de comprometimento físico, tanto no que se refere à mobilidade, pois vários eram cadeirantes, como também com relação às dificuldades na fala e na escuta que alguns participantes apresentavam. Porém, tais dificuldades não foram impeditivas para participação ativa desses idosos, ao contrário. O contato com esses sujeitos nos levou a trabalhar com suas potencialidades e possibilidades de criação.

Em um primeiro momento, com os idosos institucionalizados, optamos por trabalhar oficinas que envolvessem a senso-percepção. Assim, partimos, inicialmente, do tato como uma forma de realizar aproximações iniciais com o grupo. Dessa maneira, em dois encontros, realizamos atividades em que os idosos procuravam realizar toques com as mãos em si mesmos, em diversas partes do corpo, e também com os pés. Vários participantes possuem atrofias nas mãos, o que foi interessante de observar a tentativa de poder sentir e se descontrair (no sentido de relaxar a musculatura e também algumas inibições no contato). Também trabalhamos com a sensação de diferentes texturas e outras atividades que envolviam outros sentidos do corpo, como o paladar, a audição e o olfato.

Essas oficinas tiveram como propósito resgatar a percepção mais apurada dos sentidos, que muitas vezes ficam enrijecidos pelos hábitos, por adoecimento e até pelo processo de institucionalização. O que foi interessante perceber, também, foi o quanto os sentidos estão relacionados com a memória, pois em várias atividades, especialmente naquelas relacionadas com o paladar e o olfato, vários idosos relataram lembranças de gostos e cheiros da infância, dos alimentos preparados em família, de suas comidas preferidas.

No grupo com os idosos institucionalizados também trabalhamos temas relacionados a datas comemorativas de nosso calendário, como, por exemplo, o dia das mães. Nesse caso, propusemos resgatar, com os participantes, a condição de filho e as memórias da relação com a mãe de cada um. Assim, foram confeccionadas cartas, que foram redigidas pelos estagiários a partir das narrativas dos idosos, no caso daqueles que apresentavam dificuldades na escrita, ou feitas pelos próprios participantes da oficina. Esse trabalho da memória (BOSI, 1994) se mostrou bastante profícuo, denso e muito significativo, tanto para os idosos quanto para nós e para os estagiários, pois tivemos a oportunidade de ouvir e resgatar histórias e experiências de um tempo que muitas vezes ficam abandonadas ou esquecidas e, juntamente com os idosos, pode-se construir outros olhares e sentidos para esse legado cultural e intergeracional.

Ao longo de todo esse tempo de trabalho, foi possível realizar muitas outras atividades, que envolviam desenvolvimento de habilidades manuais, resgate de histórias de vida e outras. Também privilegiamos fazer nossos encontros não somente na sala da UNATI, mas em diversos espaços do campus, como na biblioteca, em salas de aula, no laboratório de informática e em outros espaços de sociabilidade da universidade. Entendemos que isso é muito importante para colocar os idosos em contato com a comunidade universitária, para que eles realmente se sintam parte daquele espaço e realizem trocas simbólicas e afetivas para fomentar as relações 
intergeracionais. Procuramos, ainda, ocupar outros espaços da cidade em oficinas abertas, em praças, parques, visita à exposições, especialmente para poder promover a inclusão dos idosos asilados no círculo de sociabilidades dos espaços urbanos.

\section{Conclusão}

Nossa experiência de três anos de atuação em parceria com a UNATI continua e esperamos poder prosseguir com esses projetos por muitos outros anos. O que temos notado, ao longo desse tempo, é que cada vez mais o envelhecimento se mostra como processo diverso e complexo, com diferentes facetas, seja em idosos institucionalizados, seja na terceira idade.

A diversidade nos processos de subjetivação na velhice convocam a Psicologia a estar em contato com a população idosa e a criar dispositivos de atenção, cuidado e intervenção que muitas vezes requerem a invenção de uma outra clínica, de uma perspectiva mais ampliada do que a convencional. Nesse sentido, os grupos e as oficinas se mostram como importantes dispositivos na promoção de espaços de sociabilidades e trocas.

A UNATI, para nós, é uma rara e rica oportunidade de promover processos de aprendizagens, seja com os idosos ou com os estagiários. Por meio de nossa entrada no projeto, podemos propiciar aos estudantes um contato com a população idosa e construir reflexões e práticas que certamente serão imprescindíveis para seus futuros, tanto no âmbito profissional, como também em suas vidas, tal como temos percebido. Para os idosos, institucionalizados ou da terceira idade, é a possibilidade de vivenciar espaços de sociabilidades, de transmissão de legados culturais, de reflexão, de ressignificação de histórias de vida. Dessa maneira, entendemos que, nesse complexo de relações, podemos contribuir para outros olhares, práticas e sentidos na Psicologia do Envelhecimento e também no projeto da UNATI de nosso campus.

\section{Referências}

BOSI, E. Memória e sociedade: lembranças de velhos. São Paulo: Companhia das Letras, 1994.

CAMARANO, A. A.; KANSO, S. Perspectivas de crescimento para a população brasileira: velhos e novos resultados. Texto para discussão $\mathrm{n}^{0} 1.426$. Rio de Janeiro: IPEA, 2009.

CONSELHO FEDERAL DE PSICOLOGIA. Envelhecimento e subjetividade: desafios para uma cultura de compromisso social. Brasília: CFP, 2008.

CORREA, M. R.; FRANÇA, S. A. M.; HASHIMOTO, F. Políticas públicas: a construção de imagens e sentidos para o envelhecimento. Estudos Interdisciplinares sobre o Envelhecimento, Porto Alegre, v. 15, n. 2, p. 219-238, 2010.

GRAEFF, L. O “mundo da velhice” e a cultura asilar: estudo antropológico sobre memória social e cotidiano de velhos no Asilo Padre Cacique, em Porto Alegre. Porto Alegre, 2005, 172 f. Dissertação (Mestrado em Antropologia). Instituto de Filosofia e Ciências humanas. Universidade Federal do Rio Grande do Sul, Porto Alegre, 2005.

MAIA, G. F.; CASTRO, G. D.; JORDÃO, A. B. Ampliando a clínica com idosos institucionalizados. Revista Mal-Estar e Subjetividade, Fortaleza, v.10, n. 1, p. 193210, mar. 2010.

MINAYO, M. C. S. Envelhecimento demográfico e lugar do idoso no ciclo da vida brasileira. In: TRENCH, B; ROSA, T. E. da 
C. (Orgs.). Nós e o outro: envelhecimento, reflexões, práticas e pesquisa. São Paulo: Instituto de Saúde, 2011. p. 7-17.

PICHON-RIVIÈRE, E. O processo grupal. São Paulo: Martins Fontes, 1983.

RAMOS, M. T. Plantão psicológico em instituição de longa permanência para idosos: um estudo fenomenológico. Campinas, 2012, 115 f. Dissertação (Mestrado em Psicologia). Centro de Vivências da Vida, Pós-Graduação em Psicologia. Pontifícia Universidade Católica, Campinas, 2012.

VERAS, R. País jovem com cabelos brancos. Rio de Janeiro: Relume-Dumará, 1994. 